\title{
Maceração fetal em gata: Relato de caso
}

\author{
Fetal maceration in a cat: Case reported \\ Maceración fetal: Reporte de un caso
}

Myrla Rodrigues Alcantara ORCID: https://orcid.org/0000-0001-9049-1285 Universidade Federal do Oeste da Bahia, Brasil E-mail: myrlaalcantara.medvet@gmail.com Marcos Wilker da Conceição Santos ORCID: https://orcid.org/0000-0002-6357-1236 Universidade Federal do Oeste da Bahia, Brasil E-mail: vilker.mwcs@gmail.com Jaine Mendes Lopes ORCID: https://orcid.org/0000-0001-8886-3502 Universidade Federal do Oeste da Bahia, Brasil E-mail: jainemendeslopes@gmail.com

Artur Azevedo Menezes

ORCID: https://orcid.org/0000-0001-6213-3106 Universidade Federal do Oeste da Bahia, Brasil E-mail: arturmenezes76@gmail.com

Priscila Assis Ferraz

ORCID: https://orcid.org/0000-0001-9109-744X Universidade de São Paulo, Brasil E-mail: prisferraz@gmail.com

Deusdete Conceição Gomes Junior ORCID: https://orcid.org/0000-0003-0928-0961 Universidade Federal do Oeste da Bahia, Brasil E-mail: deudete.gomes@ufob.edu.br Alexandra Soares Rodrigues ORCID: https://orcid.org/0000-0001-6821-4142 Universidade Federal do Oeste da Bahia, Brasil

E-mail: alexandra.rodrigues@ufob.edu.br

\begin{abstract}
Resumo
Objetivou-se relatar um caso de maceração fetal em uma gata, de aproximadamente 3 anos, pesando $5,0 \mathrm{~kg}$ sem raça definida, após uso de dose de contraceptivo a base de acetato de medroxiprogesterona pós cobertura. A paciente deu entrada no Hospital Veterinário Universitário da UFOB apresentando desconforto abdominal e corrimento vaginal sanguinolento fétido, com histórico de aplicação de anticoncepcional. No exame físico, apesar dos parâmetros não estarem alterados, foi notado elevada distensão abdominal bilateral. A palpação notou-se aumento de volume uterino com presença de fluido e material firme. Na ultrassonografia abdominal observou-se presença de estruturas ósseas de três fetos com silhuetas irregulares e ausência de batimentos cardíacos, constatando-se, assim, que se tratava de um quadro de morte fetal em processo de maceração. Após estabilização clínica, o animal foi submetido a ovariohisterectomia. Sete dias após o procedimento cirúrgico, houve o retorno do paciente onde se encontrava ativa, assim, sendo realizada a retirada dos pontos e alta médica.
\end{abstract}

Palavras-chave: Anticoncepcional; Distocia; Felino.

\section{Abstract}

The objective was to report a case of fetal maceration in a cat, approximately 3 years old, weighing $5.0 \mathrm{~kg}$, no defined breed, who had received a contraceptive dose of medroxyprogesterone acetate post coverage. The patient was admitted to the University Veterinary Hospital of UFOB presenting. abdominal discomfort and fetid bloody vaginal discharge, with a history of application of the contraceptive. In the physical exam, although the parameters are not altered, it was noticed a large bilateral abdominal distension. Palpation showed an increase in uterine volume with presence of fluid and firm material. In the abdominal ultrasonography, bone structures of three fetuses were observed, with irregular silhouettes, and absence of heartbeat, thus showing that it was a fetal death with a maceration process. After clinical stabilization, the animal was submitted to ovariohysterectomy. Seven days after the surgical procedure, the patient was active, thus, the removal of stitches was performed, followed by medical discharge.

Keywords: Contraceptive; Dystocia; Feline. 


\begin{abstract}
Resumen
Se tuvo como objetivo reportar un caso de maceración fetal en una gata, de aproximadamente 3 años, con un peso de $5.0 \mathrm{~kg}$ sin raza definida, a la cual se le había aplicado una dosis de anticonceptivo a base de acetato de medroxiprogesterona post cobertura. El paciente ingresó en el Hospital Universitario Veterinario de la UFOB, con malestar abdominal y flujo vaginal sanguinolento, fétido, con historial de aplicación de anticonceptivos. En el examen físico, aunque los parámetros no estaban alterados, se observó elevada distensión abdominal bilateral. En la palpación, se notó un aumento en el volumen uterino con la presencia de líquido y material firme. La ecografía abdominal mostró la presencia de estructuras óseas de tres fetos con siluetas irregulares y ausencia de latidos cardíacos, confirmando así la muerte fetal y el proceso de maceración. Después de la estabilización clínica, el animal fue sometido a una ovariohisterectomía. Siete días después del procedimiento quirúrgico, la paciente estaba activa, los puntos fueron removidos y fue dada de alta.
\end{abstract}

Palabras clave: Anticonceptivos; Distocia; Felino.

\title{
1. Introdução
}

Em sua origem o termo maceratione provém do latim, representando alterações degenerativas desintegradoras do feto, definida como processo séptico que ocorre no útero gravídico, e causa a esqueletização, liquefação e amolecimento dos tecidos moles fetais, mantendo-se apenas as estruturas ósseas (Toniollo et al., 2003; Prestes et al., 2017).

Dentre os fatores predisponentes à maceração fetal correlaciona-se o uso de contraceptivos em gatas gestantes, o qual pode manter altos níveis de progesterona (P4) circulantes por longo período de tempo no organismo do animal, o que leva a impossibilidade de contração uterina e dilatação cervical adequada, impedindo a expulsão fetal (Loretti et al., 2004; Luz et al., 2005), podendo prolongar a gestação, o que ocasiona sofrimento fetal e a morte intrauterina dos fetos com mumificação ou maceração, exigindo, assim, intervenção cirúrgica (Jericó et al., 2015).

Dentre os contraceptivos mais utilizados destaca-se o análogo sintético de progesterona que quando aplicado em fêmeas gestantes poderá causar atraso no parto e distocia com consequente retenção e maceração fetal (Monteiro et al., 2009; Montanha et al., 2012), colocando em risco a vida da fêmea (Oliveira et al, 2003; Kahn, 2011; Lichtler, 2014). Os progestágenos são os contraceptivos mais utilizados por profissionais não qualificados, favorecem inúmeros efeitos adversos, causados, principalmente, por dosagem excessiva ou administração durante estágios inadequados do ciclo estral (Bocardo et al., 2008; Dias et al., 2013).

Segundo Fernandes et al. (2020) o mecanismo de ação dos progestágenos envolve alguns processos, sendo eles a inibição dos hormônios gonadotróficos, a prevenção local do crescimento folicular ovariano e ovulação, bem como a inibição do comportamento sexual. Em fêmeas, quando aplicadas exercem feedback negativo e reduzem as concentrações de estrógeno, quando administradas na fase de anestro prevenindo o retorno do estro.

Dentre as fases do ciclo estral, as fases de proestro, estro e metaestro são contraindicados para o uso dos análogos da progesterona, bem como em fêmeas pré-púberes, obesas, diabéticas, com patologias do trato reprodutivo ou das glândulas mamárias. Podendo ser realizada a aplicação, exclusivamente em fêmeas adultas e em anestro, porém ainda com contraindicações (Oliveira et al., 2020)

Após o óbito do concepto ocorre a permanência do feto morto no útero (Prestes et al., 2017) sofrendo a ação de bactérias que desencadeiam os sinais de putrefação fetal. Estes microrganismos patogênicos podem adentrar ao útero por via ascendente a partir da cérvix (Rodrigues et al., 2018). As principais bactérias presentes nessa enfermidade são microrganismos oportunista da microbiota vaginal como a E. coli, Staphylococcus sp., Streptococcus sp. os quais ascendem ao útero e causam infecção fetal (Givens et al., 2008).

Essa afecção se dá pela liquefação e amolecimento dos tecidos moles fetais, resultando na presença estruturas ósseas no útero e exsudato purulento de odor fétido. A parede uterina apresenta-se espessa, consistente e, às vezes, intensamente fibrosada ou pode sofrer avançado grau de deterioração e ser perfurada pelas estruturas ósseas (Nascimento et al., 2003). 
Vários são os sinais clínicos em gatas, entre eles redução gradativa do apetite, emagrecimento, desconforto abdominal, corrimento vaginal de coloração variada com odor fétido, podendo estar presente neste conteúdo, fragmentos de tecidos e ossos fetais; em alguns casos pode evoluir para peritonite, devido a perfuração uterina, podendo provocar aderências, dispneia e, às vezes, hipertermia e o óbito (Toniollo et al., 2003).

O diagnóstico baseia-se no histórico, exames físicos e laboratoriais, como hemograma onde pode ser observada leucocitose ou anemia, além de exames complementares como a ultrassonografia e radiografia abdominal (Santos, 2017).

Os métodos de diagnóstico por imagem difundido nas rotinas das clínicas veterinárias são de grande auxílio quando se trata da avaliação e determinação do prognóstico de cada caso, a ultrassonografia é o exame de eleição para chegar ao diagnóstico definitivo, por permitir avaliar a viabilidade fetal (Beltrame et al., 2010). Há casos que, anamnese, sinais clínicos e exames por imagem não são suficientes para confirmação do diagnóstico, sendo necessária a realização de celiotomia exploratória, em caráter de urgência, antes que ocorra toxemia e choque (Montanha et al., 2012).

O tratamento definitivo é realizado por meio da ovariohisterectomia $(\mathrm{OH})$ terapêutica, acompanhado do tratamento clínico com antibioticoterapia de suporte (Nelson et al., 2015).

Desta forma, o presente estudo teve como objetivo relatar um caso de maceração fetal em uma gata, após uso de contraceptivo a base de acetato de medroxiprogesterona pós cobertura.

\section{Metodologia}

Foi atendido no Hospital Veterinário Universitário da Universidade Federal do Oeste da Bahia (HVU-UFOB), uma gata, fêmea, sem raça definida, de aproximadamente 3 anos, pesando 5,0 kg apresentando secreção oriunda da vagina com aspecto sanguinolento, coloração amarronzada e fétida a cerca de 24 horas (Figura 1). 
Figura 1A. Presença de corrimento sanguinolento de coloração amarronzada. 1B- Animal com presença de aumento de volume abdominal.

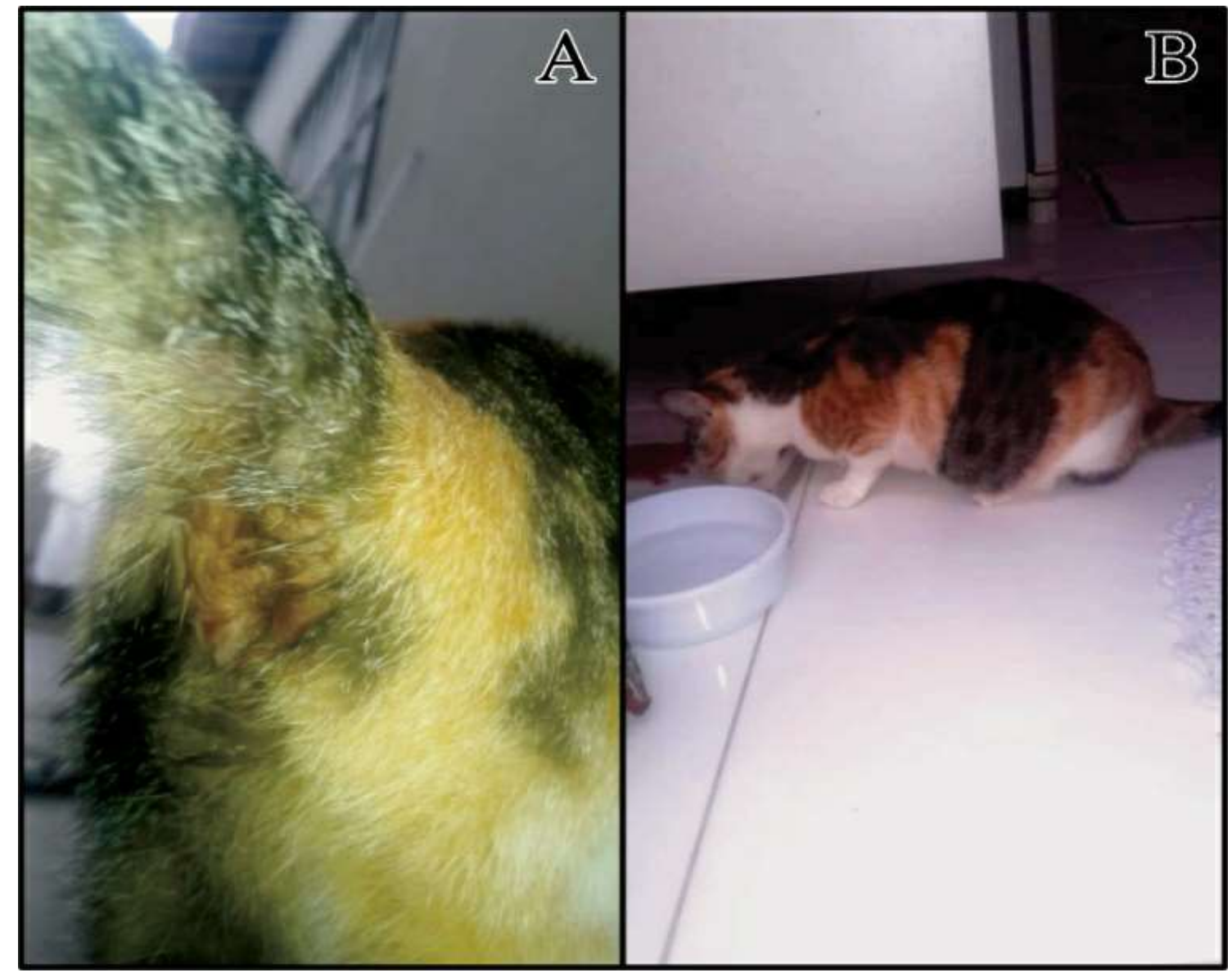

Fonte: Arquivo pessoal.

\section{Anamnese}

Durante a anamnese constatou-se que o animal possuía vacinação antirrábica, não vermifugado e sem presença de ectoparasitas, se alimentava de ração e comida caseira duas vezes ao dia, vivia em um ambiente doméstico com acesso à rua controlado e sem relato de doença anterior.

A tutora revelou que em setembro de 2019, a fêmea havia tido filhotes e em dezembro do mesmo ano, aplicou uma dose de anticoncepcional progestágeno a base de acetato de medroxiprogesterona (Promone-E ®, Pfizer Saúde Animal Zoetis, Morumbi - SP, Brasil), com o objetivo de prevenir a gestação ou promover o aborto por constatar que o animal apresentava sinais de estro, e havia copulado. Apesar da administração do contraceptivo a gestação evoluiu normalmente, no entanto, não houve sinais de parto. Aproximadamente 3 meses após a aplicação do progestágeno, observou-se que a gata estava quieta, sem apetite e tomando água normalmente, dias depois notou secreção oriunda da vagina com aspecto sanguinolento, coloração amarronzada e fétida, buscando então atendimento médico veterinário.

\section{Achados de exame físico}

No exame físico foi notada atitude normal, linfonodos sem reatividade, mucosas normocoradas, discreta desidratação, tempo de preenchimento capilar (TPC) de 2 segundos, frequência cardíaca (FC) de 180bpm e respiratória (FR) de 32mpm, temperatura retal (TR) de $38,5^{\circ} \mathrm{C}$, pulso forte e sincrônico. 
Notou-se elevada distensão abdominal bilateral. O animal demonstrou desconforto á palpação abdominal, abdome tenso com nítido aumento de volume uterino com presença de fluido e áreas firmes, mas não foram notados movimentos fetais. Não foi possível auscultar batimentos cardíacos fetais. Não foram identificadas alterações na pelve como estreitamento, lesões ou fraturas. As glândulas mamárias apresentavam-se simétricas, sem alterações, discretamente edemaciada e sem secreção. Após inspeção e palpação da vulva foi observado as seguintes alterações: secreção vulvo-vaginal sanguinolenta de coloração amarronzada, consistência aquosa e odor fétido.

\section{Auxiliar de diagnóstico}

O exame de ultrassonografia abdominal revelou cornos uterinos com parede espessada e distendidos às custas de presença de conteúdo hipoecóico, notou-se também presença estruturas ósseas de aproximadamente 3 fetos com silhuetas irregulares e ausência de batimentos cardíacos, constatando-se assim morte fetal em processo maceração (Figuras 2 e 3 ).

Figura 2. Imagem ultrassonográfica do abdômen evidenciando a presença de calota craniana de um dos fetos no útero.

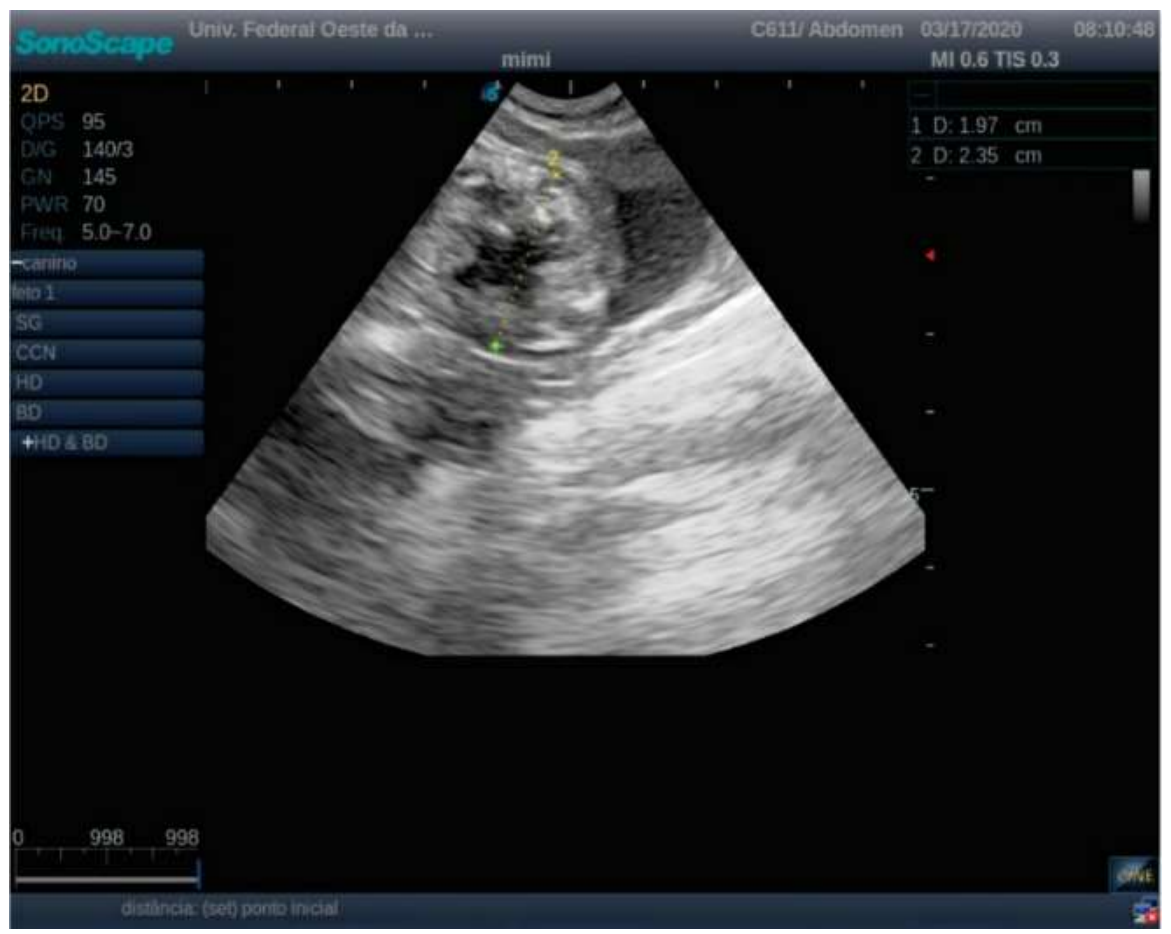

Fonte: Setor de Diagnóstico por Imagem do HVU- UFOB. 
Figura 3. Imagem ultrassonográfica do abdômen evidenciando o tórax de um dos fetos com ausência de batimentos cardíacos.

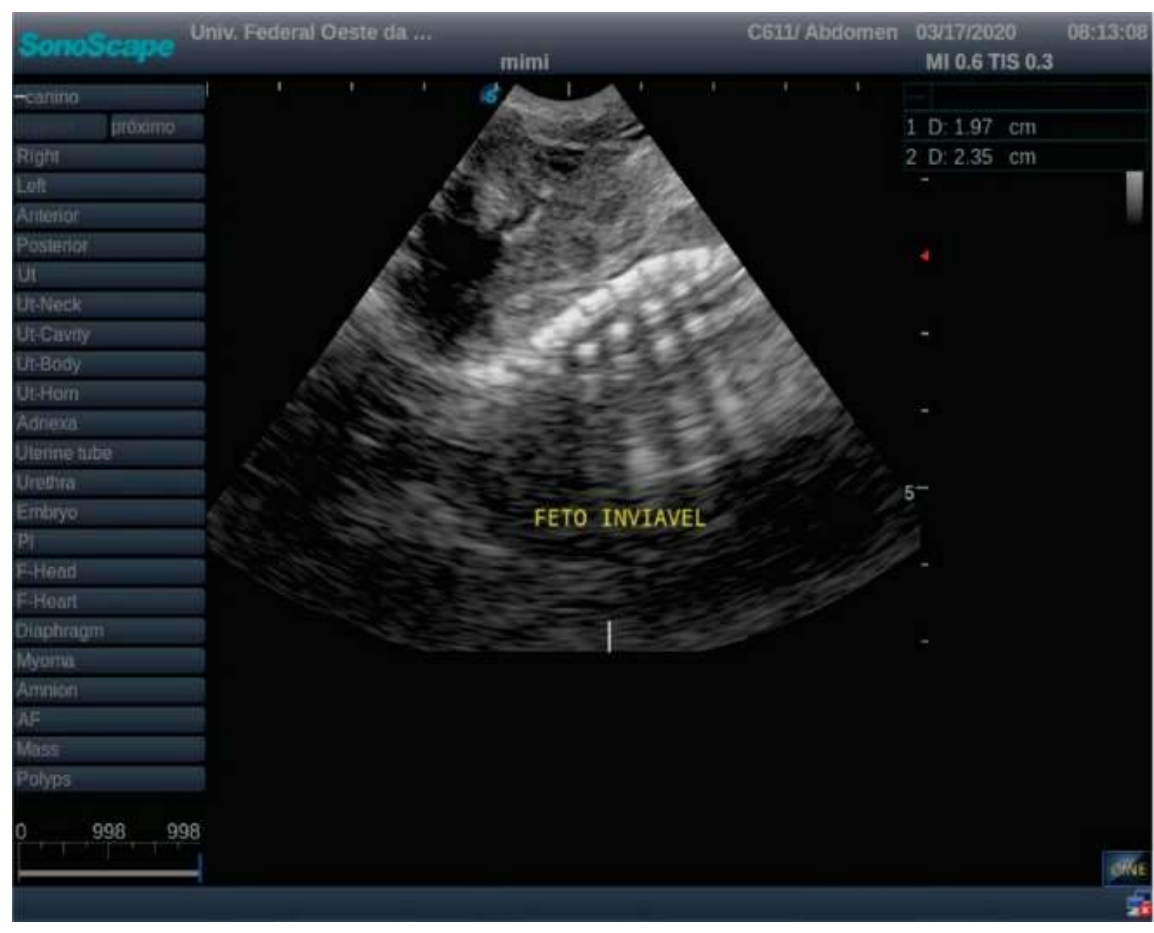

Fonte: Setor de Diagnóstico por Imagem do HVU- UFOB.

\section{Tratamento}

Diante do diagnóstico de maceração fetal e considerando a gravidade e o estado geral de saúde da paciente optou-se por estabilização com fluidoterapia com solução fisiológica de cloreto de sódio $0,9 \%$, seguida da OH terapêutica. Na avaliação pré-cirúrgica da fêmea um dia após o atendimento clínico, mensurou-se: FC: 240bpm, FR: 20mpm, TPC: 2 segundos e TR: 38,5 C $\mathrm{C}^{\mathrm{o}}$, mucosas normocoradas e hidratação moderada. Com base no histórico e informações clínicas, a paciente foi classificada quanto ao estado físico e risco anestésico em ASA III (animal com doença sistêmica grave). O animal foi preparado para procedimento cirúrgico sendo realizada tricotomia ampla do abdômen desde a cartilagem xifoide até o púbis com posterior assepsia abdominal com solução alcoólica de Digluconato de Clorexidina (Riohex 0,5\%®, RioQuímica S/A., São José do Rio Preto - SP, Brasil). Como medicação pré-anestésica (MPA) foram administrados Clorpromazina (Amplictilß, Cristália, Produtos Farmacêuticos Ltda., Itapira - SP, Brasil) 1mg/kg, via subcutânea; Morfina (Dimorf®, Cristália, Produtos Farmacéuticos Ltda., Itapira - SP, Brasil) na dose $0,1 \mathrm{mg} / \mathrm{kg}$, via intramuscular; e Dipirona (Dipirona sódica® $500 \mathrm{mg} / \mathrm{ml}$, Halex Istar Indústria Farmacêutica Ltda. Goiânia - Go, Brasil) na dose 25mg/kg, via subcutânea. Para indução da anestesia geral foram administrados Propofol (Propovan®, Cristália, Produtos Farmacêuticos Ltda, Itapira - SP, Brasil) na dose 5mg/kg, via intravenosa; Cetamina (Ketalar@ Instituto Biochimico Indústria Farmacêutica Ltda, Itatiaia - RJ, Brasil) na dose 1mg/kg na via intravenosa; e para manutenção da anestesia geral inalatória foi utilizado isoflurano em circuito baraka.

Após a colocação dos panos de campo seguiu-se a celiotomia habitual. Durante o acesso observou-se o útero da gata com aumento de volume nos cornos uterinos e grande vascularização (Figura 4). 
Figura 4. Útero com aumento de volume nos cornos uterinos e presença de grande vascularização.

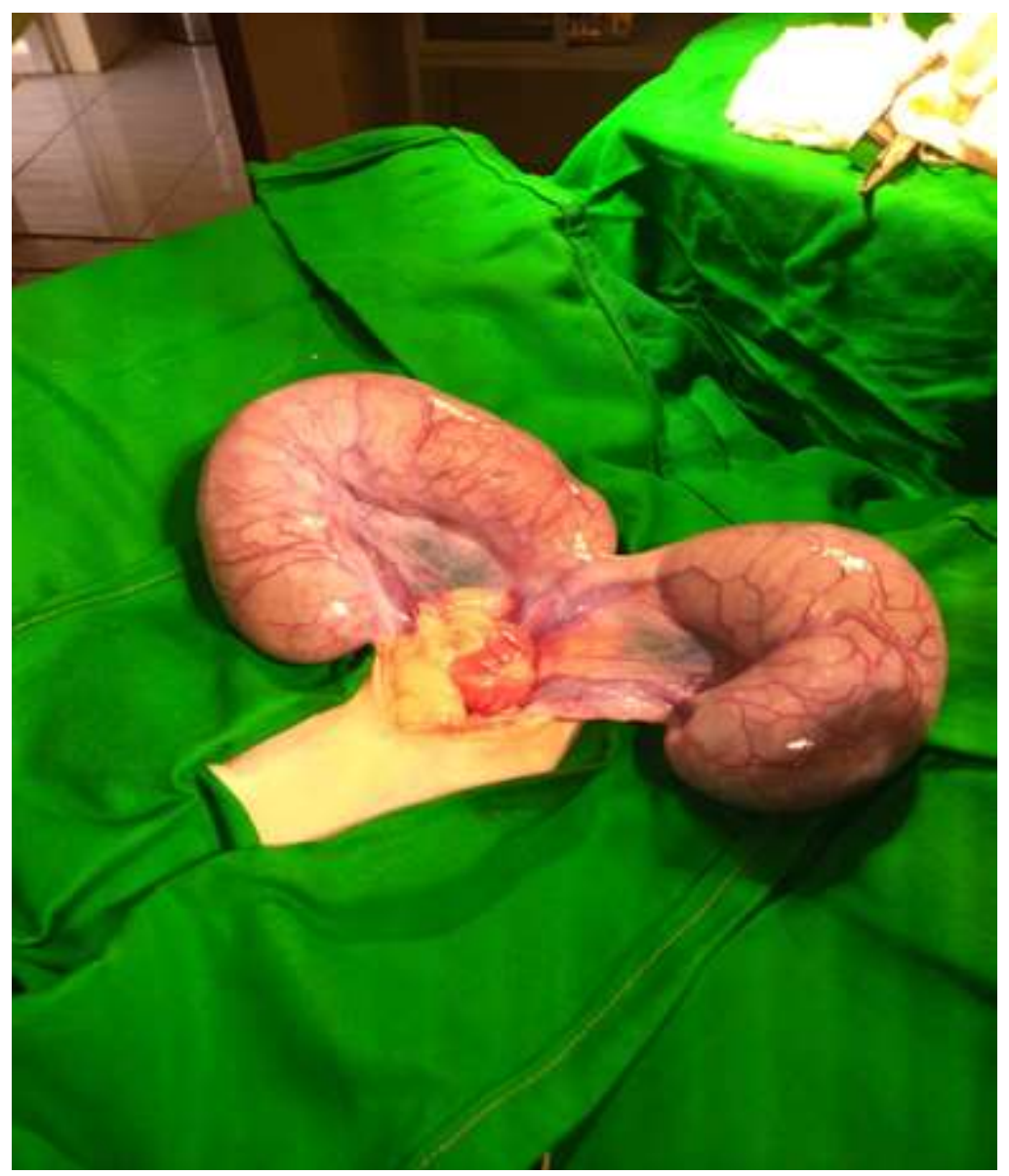

Fonte: Setor de Cirurgia do HVU- UFOB.

\section{Achado macroscópicos}

Após exposição uterina observou-se que, macroscopicamente, os ovários e tubas uterinas não apresentavam alterações (Figura 5), enquanto o útero se mostrava bem estruturado e consistente, com vasos ingurgitados (Figura 6 e 7). Finalizada a $\mathrm{OH}$ o paciente recebeu os cuidados com a ferida e colocação de roupa cirúrgica bem como avaliação de recuperação anestésica e foi direcionado a sala de pós-operatório. O material coletado foi direcionado ao setor de patologia animal do HVU-UFOB onde após abertura, observou-se no lúmen uterino fetos macerados com ossos em esqueletização desorganizada e tecidos materno-fetais liquefeitos envoltos em exsudato mucopurulento e fétido (Figura 8). 
Research, Society and Development, v. 10, n. 7, e23710716422, 2021

(CC BY 4.0) | ISSN 2525-3409 | DOI: http://dx.doi.org/10.33448/rsd-v10i7.16422

Figura 5. Ovário da fêmea com aparência normal.

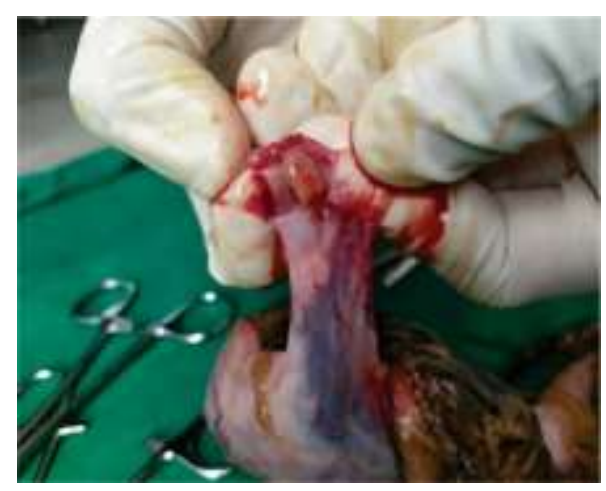

Fonte: Setor de Patologia Veterinária do HVU-UFOB.

Figura 6. Corno uterino direito com, aproximadamente, $22 \mathrm{~cm}$ de comprimento.
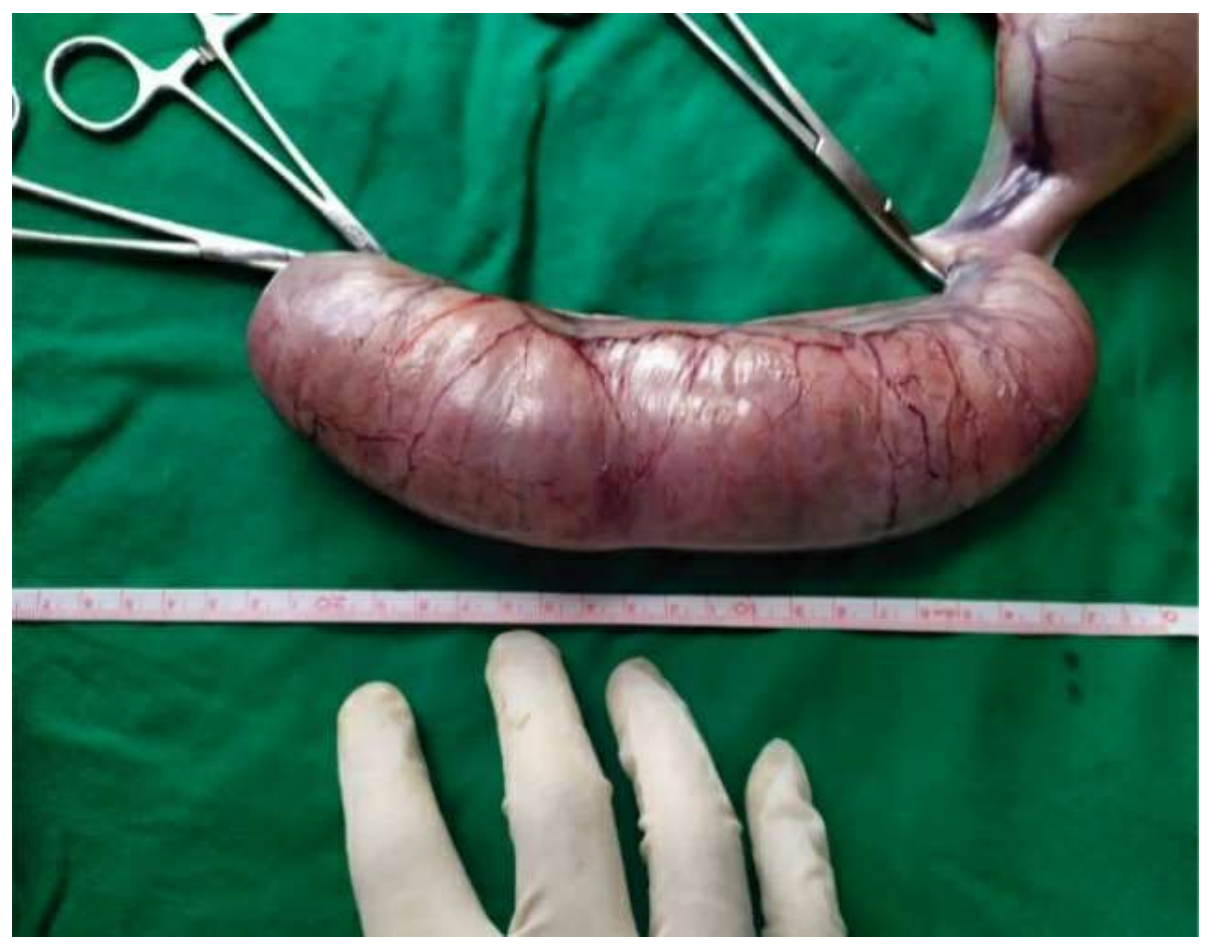

Fonte: Setor de Patologia Veterinária do HVU-UFOB. 
Research, Society and Development, v. 10, n. 7, e23710716422, 2021

(CC BY 4.0) | ISSN 2525-3409 | DOI: http://dx.doi.org/10.33448/rsd-v10i7.16422

Figura 7. Corno uterino esquerdo com, aproximadamente, $10 \mathrm{~cm}$ de comprimento.

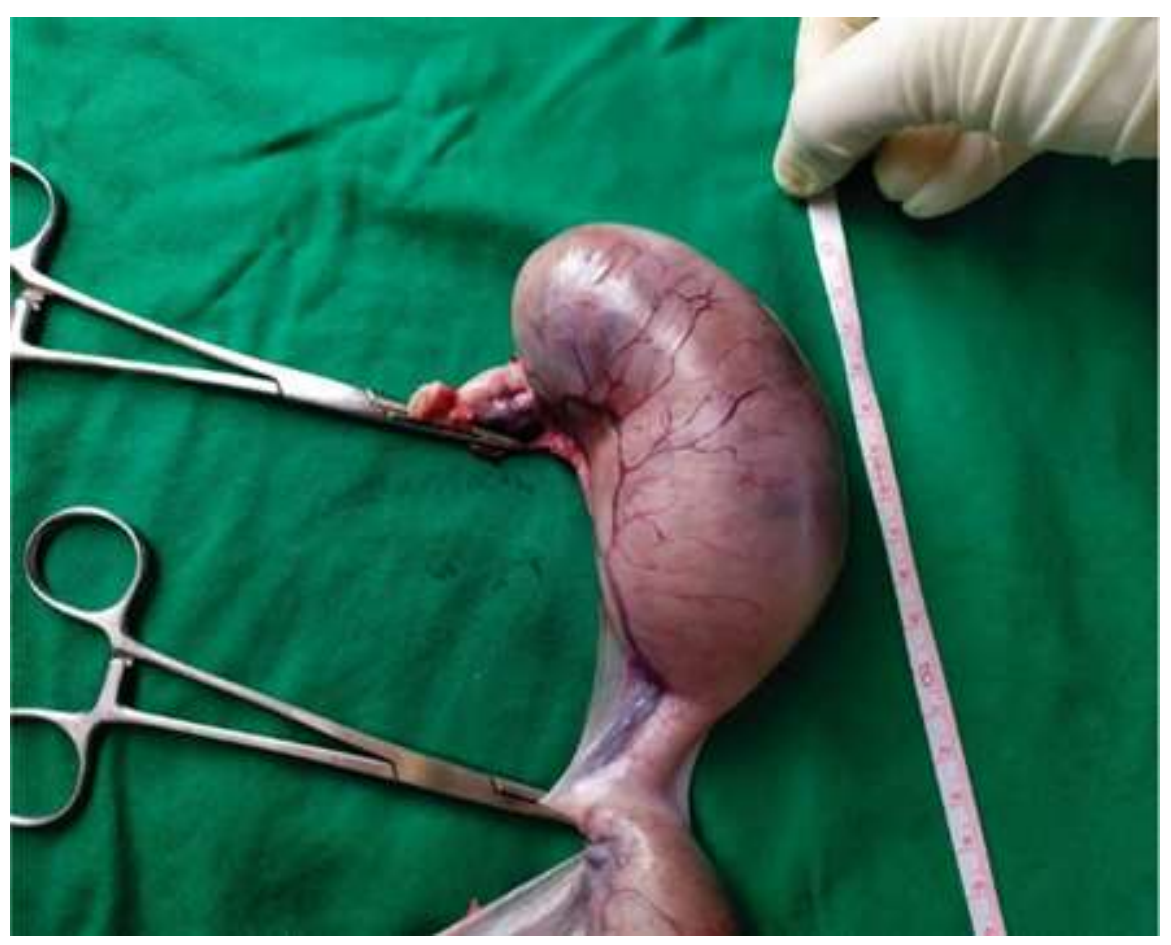

Fonte: Setor de Patologia Veterinária do HVU-UFOB.

Figura 8. Cornos uterinos abertos revelando 3 fetos felinos macerados e disformes.

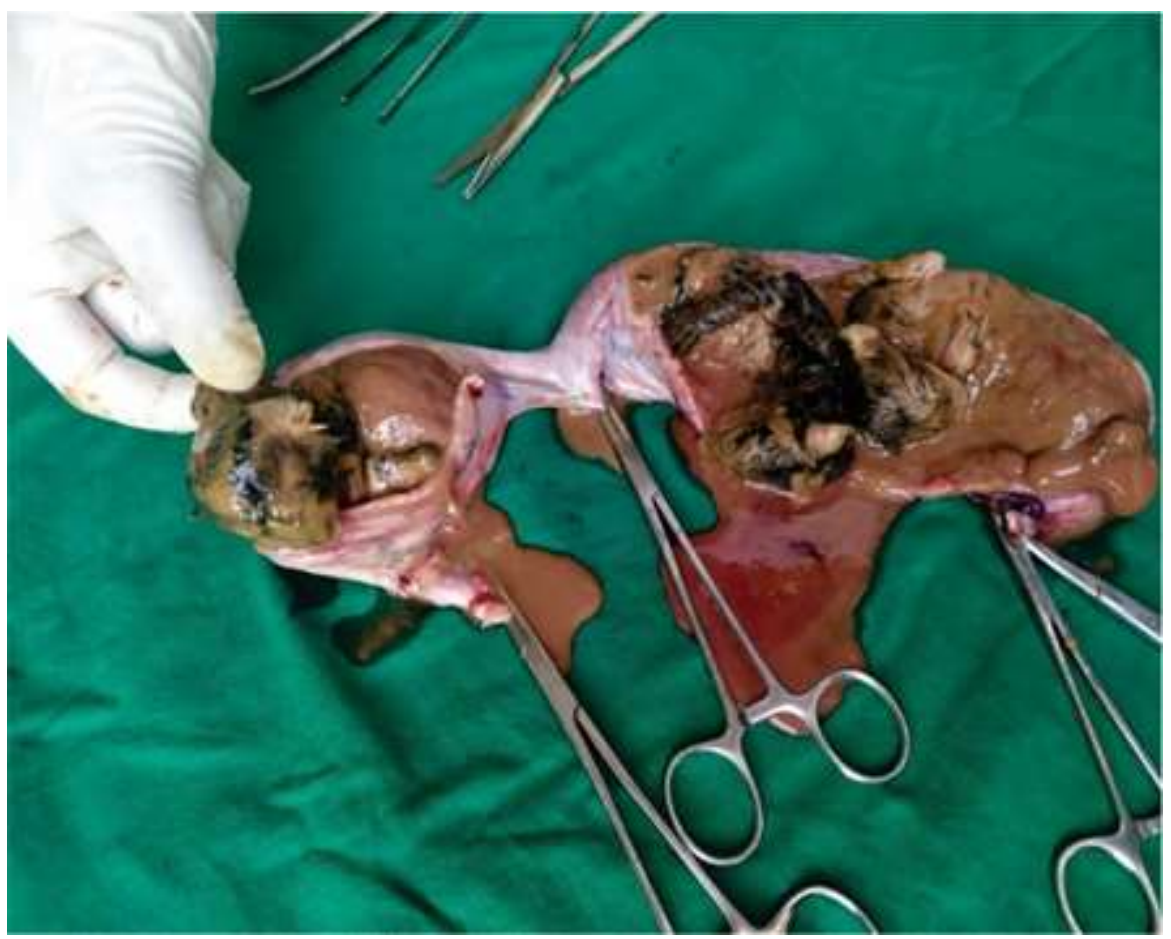

Fonte: Setor de Patologia Veterinária do HVU-UFOB. 


\section{Evolução}

Após a recuperação anestésica o paciente foi liberado para acompanhamento domiciliar, sendo solicitada a utilização de roupa cirúrgica em tempo integral e prescrito Amoxicilina triidratada, na dose 0,1mg/kg, via intramuscular, no dia da cirurgia e mais duas aplicações com intervalo de $48 \mathrm{~h}$ entre elas Meloxicam na dose $0,1 \mathrm{mg} / \mathrm{kg}$, via subcutânea; no dia da cirurgia e após mais duas aplicações com intervalo de 24 horas entre elas, e foi recomendado o uso de dipirona $25 \mathrm{mg} / \mathrm{kg}$, via oral, uma vez ao dia, caso a fêmea apresentasse sinais de dor e inquietação. O proprietário foi contatado diariamente e revelou que a gata voltou a se alimentar espontaneamente em menos de 24 horas. Sete dias após o procedimento cirúrgico o animal apresentava-se hígido e sem alterações na cicatrização, sendo removido os pontos e dada alta médica.

\section{Discussão}

Ao longo da anamnese a tutora afirmou que promoveu a aplicação de um fármaco a base de acetato de medroxiprogesterona, após a cobertura, corroborando aos achados de Montanha et al. (2012) em caso semelhante de maceração fetal em uma fêmea felina, após uso de contraceptivo três meses antes do início dos sinais clínicos.

A mesma relatou ainda que fez o uso do medicamento na tentativa de evitar a gestação ou promover o aborto, porém este método se fez ineficaz como demonstra González-Domínguez e Maldonado-Estrada (2006), os quais afirmaram que quando progestágenos sintéticos, como o acetato de medroxiprogesterona e proligestona, são administrados para evitar os efeitos da cópula indesejada, estes medicamentos não foram eficientes na prevenção da prenhez, pois o proestro e o estro já seguiram seu curso clínico.

Os parâmetros fisiológicos da fêmea ao exame se encontravam dentro da normalidade e as alterações comportamentais foram lentamente percebidas pela proprietária, fato este que pode ocorrer quando o animal está apresentando a forma silenciosa da patologia, como foi evidenciado por Santos (2017), contrariando a previsão de ocorrência de hipertermia associado a infecção conforme descrito por Toniollo et al. (2003).

Em relação aos sinais clínicos, houve aumento de volume abdominal, a fêmea demonstrou desconforto na palpação abdominal que se apresentava firme e tenso, além da perda de apetite. O sangramento vaginal de coloração amarronzada e de odor pútrido neste caso, bem como os sinais supracitados condizem com a literatura, pois de acordo a Toniollo et al. (2003) dentre os sinais clínicos da maceração fetal, pode estar presente corrimento vaginal de coloração variada e com odor fétido, desconforto abdominal e perda gradativa do apetite. Além disso, Nascimento e Santos (2000) cita ocorrência de exsudato purulento de odor fétido, que eventualmente, é aquoso e sem odor.

Constatou-se no presente caso que a fêmea teve a gestação prolongada, evoluindo para morte e maceração fetal intrauterina, em decorrência do uso de medicamento hormonal a base de acetato de medroxiprogesterona. As alterações fetais foram observadas, após abertura uterina extra-abdominal com a presença de três fetos em processo de autólise dos tecidos moles, evidenciando a perda da integridade e silhueta corporal dos mesmos.

A prorrogação da gestação observado neste trabalho, ocasionou a morte e não expulsão dos fetos, isto pode ser explicado pelo fato da progesterona exógena administrada antes ou durante a gestação não ocasionar o aborto imediato, porém a mesma permanece em níveis elevados na corrente sanguínea até o momento do trabalho de parto, impedindo o seu desenvolvimento, por inibir o aumento de estrógeno, ocitocina e prostaglandina F2 $\alpha$.

Para ocorrência do parto, o ambiente endócrino materno deve sofrer alterações, em que o evento da maturação fetal exerce papel fundamental no início deste processo com a liberação de cortisol fetal que depende do decréscimo das concentrações plasmáticas de P4 e aumento de estrógenos, bem como produção de prostaglandinas para seguir o parto normalmente, como o decréscimo de P4 não acontece o evento se torna inviável, como relatou Luz et al. (2005) ao abordar a ocorrência de distocia por fator hormonal exógeno em cadelas. 
Devemos atentar que a paciente sofreu efeito adverso devido a utilização indiscriminada de contraceptivo sem assistência profissional adequada, o mesmo foi demonstrado por Dias et al. (2013) em seu trabalho ao questionar 100 proprietários sobre o uso, frequência, forma de aplicação e nível de assistência, concluiu-se que $92 \%$ dos entrevistados faziam uso indiscriminado e inadvertidos, sem orientação médica veterinária de fármacos contraceptivos em cães e gatos, justificando assim o alto número de animais que sofreram quadros patológicos associados à aplicação.

De acordo com Fernandes et al. (2020) os progestágenos e estrógenos são os contraceptivos mais utilizados e aplicados por profissionais não qualificados, favorecendo assim os inúmeros efeitos adversos, estes, portanto são provavelmente causados por dosagem excessiva e/ou administrados durante outros estágios do ciclo estral que não o anestro.

Neste trabalho o animal foi submetido à cirurgia $\mathrm{OH}$ de urgência, como recomenda a literatura sendo confirmado o quadro de maceração fetal após o procedimento, assim também como descreveu Bolson et al. (2004) e Montanha et al. (2012) uma vez que há casos onde se faz necessária a celiotomia exploratória, realizada em caráter de urgência, antes que ocorra toxemia e choque, alterações estas não observada na paciente.

Neste caso não houveram complicações intra e pós-operatórias, a recuperação pós-anestésica-cirúrgica da fêmea foi satisfatória, levando em consideração o diagnóstico preciso, impediu-se assim que os efeitos sistêmicos da infecção no útero fossem prolongados. Neste sentido a escolha dos antibióticos de amplo espectro, fluidoterapia de suporte e anti-inflamatório não esteroidal pré e pós-operatórios exerceram eficácia desejada. A paciente voltou a se alimentar imediatamente após a recuperação anestésica, recebeu os devidos cuidados com a ferida cirúrgica bem como repouso, o que culminou em rápida cicatrização e boa aparência, recebendo a alta médica, sete dias após o procedimento quando os pontos foram removidos.

É importante salientar que nem todos os casos de intervenção cirúrgica obtém-se o sucesso, especialmente quando há demora no diagnóstico e tratamento, como foi o caso relatado por Gomes et al. (2020) em que o mesmo constatou prognóstico desfavorável de maceração, onde houve comprometimento de vários órgãos na cavidade abdominal e pelo difícil retorno no pós-operatório, a conduta médica optou-se pela eutanásia.

\section{Considerações Finais}

O uso de fármacos anticoncepcionais são contraindicados para as gatas nas fases do ciclo estral, como proestro, estro e diestro, podendo provocar a ocorrência de prolongamento da gestação com consequente maceração fetal pela deficiente dilatação da cérvix e os altos níveis séricos de progesterona no momento do parto, conforme observado no paciente desse relato. O sucesso do tratamento é proporcional a rapidez de diagnóstico e instituição de terapia adequada.

\section{Referências}

Balthazar da Silveira, C. P., Machado, E. A. A., Silva, W. M., Marinho, T. C. M. S., Ferreira, A. R. A., Bürger, C. P., \& Costa Neto, J. M. D. (2013). Estudo retrospectivo de ovariossalpingo-histerectomia em cadelas e gatas atendidas em Hospital Veterinário Escola no período de um ano. Arquivo Brasileiro de Medicina Veterinária e Zootecnia, 65(2), 335-340.

Beltrame, R. T., Fernandes, D. R., Quirino, C. R., Machado, F. M., \& Saraiva, C. (2010). Aplicações da ultra-sonografia na obstetrícia animal. Pubvet, 4, Art893.

Bocardo, M., Dabus, D. M. M., Tentrin, T. D. C., Lima, G. D. S., \& Bariani, M. H. (2008). Influência hormonal na carcinogênese mamária em cadelas. Revista Científica Eletrônica de Medicina Veterinária, 6(11), 1-6.

Bolson, J., de Godoy, C. B., Ornes, R. C., Schossler, J. E. W., \& Pachaly, J. R. (2004). Fisometra em cadela (Canis familiaris Linnaeus, 1758) - relato de caso. Arquivos de Ciências Veterinárias e Zoologia da UNIPAR, 7(2).

Dias, L. G., Oliveira, M. É., Dias, F., Calazans, S., \& Conforti, V. (2013). Uso de fármacos contraceptivos e seus efeitos adversos em pequenos animais. Enciclopédia Biosfera, 9(16).

Fernandes, E. R. L., Costa, T. M., \& Leite, D. F. S. S. (2020). Uso de fármacos contraceptivos e seus efeitos colaterais em cães e gato: Revisão de literatura. Revista de Medicina VeterrináriaVII, 17(23), 1-14.

Givens, M. D., \& Marley, M. S. D. (2008). Infectious causes of embryonic and fetal mortality. Theriogenology, 70(3), $270-285$. 
Research, Society and Development, v. 10, n. 7, e23710716422, 2021

(CC BY 4.0) | ISSN 2525-3409 | DOI: http://dx.doi.org/10.33448/rsd-v10i7.16422

Gomes, R. S. D. S., Colpani, A., De Almeida, F. A. B. I. O., \& Queiroz, S. (2020). Maceração Fetal Em Felino-Relato De Caso. Revista Scientia Rural-ISSN $2178-3608,1$.

González, M. S., \& Estrada, J. G. M. (2006). Gestación prolongada asociada con la prescripción inadecuada de medroxiprogesterona acetato.¿ Es racional y ético el uso de progestágenos exógenos en perras?. Revista Colombiana de Ciencias Pecuarias, 19(4), 442-450.

Jericó, M. M., Kogika, M. M., \& de Andrade Neto, J. P. (2015). Tratado de medicina interna de cães e gatos. Grupo Gen-Guanabara Koogan.

Kahn, C. M (2011). . Manual Merck: Saúde Animal. Editora Roca.

Lichtler, J. (2014). Castração precoce em pequenos animais: técnica, vantagens e riscos e uso no controle populacional.

Loretti, A. P., Ilha, M. R. S., Breitsameter, I., \& Faraco, C. S. (2004). Clinical and pathological study of feline mammary fibroadenomatous change associated with depot medroxyprogesterone acetate therapy. Arquivo Brasileiro de Medicina Veterinária e Zootecnia, 56(2), 270-274.

Montanha, F. P., Corrêa, C. D. S., \& Parra, T. C. (2012). Maceração fetal em gata em decorrência do uso de contraceptivos-relato de caso. Revista Científica Eletrônica de Medicina Veterinária, 10(9), 1-6.

Monteiro, C. M. R., Perri, S. H. V., Carvalho, R. G. D., \& Koivisto, M. B. (2009). Histologia e morfometria em cornos uterinos de cadelas nulíparas, multíparas e tratadas com contraceptivos. Pesquisa Veterinária Brasileira, 29(10), 847-851.

Nascimento, E. F., \& de Lima Santos, R. (2000). Patologia Da Reprodução Dos Animais Domésticos. Grupo Gen-Guanabara Koogan.

Nelson, R., \& Couto, C. G. (2015). Medicina interna de pequenos animais. Elsevier Brasil.

Oliveira Rédua, C. R., \& Bueno, L. C. V. (2020). Uso E Consequências Dos Principais Métodos Contraceptivos Em Cadelas Na Região Do Distrito Federal. Revista Ciência e Saúde Animal, 2(1).

Oliveira, E. C. S., Marques, A. P., \& Neves, M. M. (2003). Endocrinologia reprodutiva e controle da fertilidade da cadela-revisão. Archives of Veterinary Science, $8(1)$

Prestes, N. C., \& da Cruz Landim-Alvarenga, F. (2017). Obstetrícia veterinária. Grupo Gen-Guanabara Koogan.

Rezende, M., Coletto, P. M., \& Zacché, E. (2005). Gestação e parto em cadelas: fisiologia, diagnóstico de gestação e tratamento das distocias. Revista Brasileira Reprodução Animal, 29, 142-150.

Rezende, M., Coletto, P. M., \& Zacché, E. (2005). Gestação e parto em cadelas: fisiologia, diagnóstico de gestação e tratamento das distocias. Revista Brasileira Reprodução Animal, 29, 142-150.

Rodrigues, J. B., Cordeiro, M. L. L., Pereira, A. G., Leite, M., de Carvalho, S. M. R., \& da Silva, T. S. (2018). Maceração fetal em cadela. Ci. Anim., 53-55.

Santos, C. V. S. (2017). Estudo retrospectivo dos aspectos radiográficos e ultrassonográficos de morte e retenção fetal em pequenos animais.

Toniollo, G. H., \& Vicente, W. R. R. (2003). Manual de obstetrícia veterinária. Varela.

Viana, D. C., dos Santos, A. C., Rui, L. A., de Oliveira, D. M., Silva, A. B., Costa, F. D. C. F. C., \& de Assís Neto, A. C. (2012). Hiperplasia Mamã Ria Felina-Relato De Caso. Veterinária Notícias, 18(2). 